\title{
REGIONALISMUS UND KULTURELLE IDENTITÄT IN ITALIEN ZWISCHEN DEM ENDE DES 19. UND DEM ANFANG DES 20. JAHRHUNDERTS: DAS BEISPIEL DER ROMAGNA UND DIE NATIONALE LAGE ${ }^{1}$
}

\author{
Stefano CAVAZZA \\ Lehrstuhl für Politologie, Institut für Geschichte, Politikwissenschaftliche Fakultät \\ I-40129 Bologna, strada Maggiore 45, Italien
}

In letzter Zeit wird das Problem des italienischen nationalen Bewußtseins viel diskutiert. ${ }^{2}$ Der jüngste erfolgreiche Aufstieg von regionalistischen Bewegungen, d. h. der sogenannten Leghe, die ihre gemeinsame nordische Identität dem italienischen Staat gegenüberstellen, haben Journalisten und Akademiker dazu gebracht, wieder auf die schwache Nationalisierung der Italiener zu verweisen. Der 1867 von Massimo D'Azeglio geäußerte Satz: „Italien ist geschaffen, aber leider schafft man nicht die Italiener" klingt noch immer aktuell. ${ }^{3}$

In der Geschichte Italiens sind solche kritischen Urteile mit echter RegelmäBigkeit immer wieder aufgetaucht. Wir können zwei verschiedene Interpretationsmuster der Situation erkennen. Eines führt die schwache nationale Identität auf das Weiterbestehen eines lokalpatriotischen Geistes, das andere auf die von oben verabsäumte Anerkennung der regionalen Vielfalt des Landes durch die Bildung eines zentralistischen Staates zurück.

Beide Sichtweisen aber teilen die Ansicht, daß es regionale Charaktere gibt und diese sehr alt und von Natur aus gegeben seien. Der „Region“-Begriff bleibt dabei aber schwer zu fassen. Im allgemeinen fällt es - obwohl wir naturgemäß homogenene Regionen mittels geographischer, wirtschaftlicher oder sprachlicher Kriterien abgrenzen können - schwer, eine Zone zu finden, die alle diese Kriterien erfüllt. ${ }^{4}$ Denken wir nämlich an das Identitätsproblem, meinen wir ein kulturelles Feld, das zwar das Ergebnis von materiellen, wirtschaftlichen und soziopolitischen Prozessen ist, aber eben eines Konstruktionsprozesses bedarf. Zur mailändischen Identität gehört etwa nicht nur die Verbundenheit zur Heimatstadt, sondern auch der Mythos, daß Mailand die ethisch-moralische Hauptstadt Italiens sei und daß die Mailänder

${ }^{1}$ Ich bedanke Bernard Tschofen der den Beitrag sprachlich bearbeitet hat.

${ }^{2}$ S. zusamenfassend CAVAZZA, Stefano: Identità e culture regionali nella storia d'Italia, in „Memoria e Ricerca“ H. 6. (Dez. 1995), pp. 51-71.

${ }^{3}$ D'AZEGLIO, Massimo: I miei ricordi. Bd. I, Florenz 1867, S. 7. Der Satz ist übrigens in der Geschichte seiner Rezeption - schon als Folge des italienischen Nationalismus - in eine Aufforderung gewandelt worden und übrigens fälschlich Cavour zugeschrieben worden: „Italien ist geschaffen. Jetzt müssen wir die Italiener schaffen!“. Dazu auch PETERSEN, Jens: Quo vadis, Italia. Ein Staat in der Krise. München 1995, S. 34.

${ }^{4}$ Stichwort: Regione. [M. RONCAYOLO], In: Enciclopedia, Bd. 11. Turin 1980, S. 772-795. 
leistungsfähiger als andere Staatsbürger seien. Die Zuschreibung dieser angeblichen Qualitäten der Mailänder ist das Ergebnis ideologischer Prozesse, in deren Verlauf und Folge sich um Mythen, Werte und Stereotypen eine Identität konstruierte. Dasselbe gilt auch für die regionale Ebene: Denkt man an die Stereotypen vom heiligen, kämpferischen und grünen Umbrien, stellt man fest, daß sie im neunzehnten Jahrhundert gebildet worden waren.

Heutzutage gibt es in Italien 20 Regionen; sie sind erst 1970 als Verwaltungsorgane etabliert worden. Aber historisch wurzeln sie oft sehr tief, und einige Bezeichnungen lassen sich schon in römischer Zeit finden. Das bedeutet aber weder, daß eine wirkliche Kontinuität vorliegt, noch, daß die Regionalisierung über längere Zeit gleichmäßig ablief und argumentierte. Im Gegenteil: Die regionalen Identitäten sind als junge und jüngere Bildungen zu verstehen. Die Basis dieses Regionalisierungsprozesses liegt freilich im neunzehnten Jahrhundert, aber als entscheidender Ausgangspunkt für das Regionalproblem ist das Vereinigungsjahr $1861 \mathrm{zu}$ verstehen. Das bedeutet nicht, daß ältere Traditionen sowie das Vorhandensein italienischer Staaten vor der Vereinigung keine Rolle gespielt hätten. Dabei muß man aber bedenken, daß diese Staaten oft nicht mit der späteren regionalen Einteilung übereinstimmten. Das sogenannte Regno delle Due Sicilie - „Königreich beider Sizilien“ - schloß das heutige Kampanien, Lucanien, Sizilien, die Abruzzen und Molise sowie Kalabrien ein. Zum Reich des Papstes, zum Kirchenstaat, gehörten Umbrien, die Emilia-Romagna und Latien. Die toskanische Identität, die sich doch sehr der literarischen Tradition und dem Toskanischen Großherzogtum verdankte, ist auf die entscheidende Wirkung von intellektuellen Gruppen und auf soziale Prozesse nach der Einigung zurückzuführen. Auf eine jüngere Basis stützt sich zum Beispiel die Identität von Umbrien, dessen Grenzen erst in den zwanziger Jahren dieses Jahrhunderts klar festgelegt wurden, und sogar in der Verfassungsdebatte nach dem Zweiten Weltkrieg wieder in Frage gestellt worden sind. ${ }^{5}$ Sehr schwach ist die Identität von Latien, wo die zentralisierenden Kräfte der Haupstadt Rom die Ausbildung eines regionalen Bewußtseins verhinderte.

Außerdem sind einige Faktoren nicht zu übersehen, die für die Bildung solcher regionaler Identitäten hinderlich waren. Erstens zidelte die italienische Politik auf eine gemeinsame Kultur und betrachtete daher die Regionen als Gefahr für die junge nationale Einheit. Das bedeutete etwa, daß die Absicht, regionale Verwaltungsebenen einzurichten, lange abgelehnt worden ist. Zweitens spielte auch die nicht zu unterschätzende Bedeutung der Stadt in der Geschichte Italiens eine Rolle. Schon 1858 hob Carlo Cattaneo in seinem Buch, Memorie istoriche di Lombardia hervor, daß das regionale Bewußtsein der ländlichen Bevölkerung weniger ausgeprägt sei als das der Städte. ${ }^{6}$ Der andauernde städtische Polyzentrismus beeinflußte den neuen Staat mächtig und behinderte die Bildung von größeren Zusammenschlüssen. Drittens verwirklichten die Regierenden trotz der Übernahme des franzö-

${ }^{5}$ BONORA, Paola: I geografi nel dibattito sulla questione regionale (1944-1948). Bologna 1980. 101.

${ }^{6}$ CATTANEO, Carlo: La città nella storia d'Italia. In RoMAGNOSI, Giandomenico-CATTANEO, CarloFERRARI, Giuseppe: Opere. (Hrsg. von Emilio SESTAN) Milano-Napoli 1962, S. 1001. 
sischen Staatsideals einen Zentralismus, der mehr ein Übereinkommen zwischen zentralen und peripheren Eliten als eine wirkliche Homogenisierung des Landes darstellte. Das heißt, daß die lokalen Machtstrukturen unverändert bis in die Zeit des Faschismus erhalten blieben. Das wurde bereits von den Zeitgenossen erkannt. 1882 etwa prangerte Pasquale Turiello den Fortbestand von lokalen Machteliten an. ${ }^{7}$ In letzter Zeit haben historische Forschungen, wie die Fallstudie Gribaudis über Eboli oder die Untersuchungen Romanellis das klar bewiesen. ${ }^{8}$

Seit den achtziger Jahren des letzten Jahrhunderts war aber ein neues Interesse für die lokale Ebene erwacht. Im literarischen Bereich lieferten Schriftsteller wie Giovanni Verga oder Grazia Deledda regionale Schilderungen, die sich zum Teil auch auf die Kenntnis volkskundlicher Materialien stützten. Die Sammlung von Überresten der Bauernkultur durch Gelehrte trug mit zur Definition der regionalen Identitäten bei. Die Volkskundler hingegen hoben die Differenzen zwischen den verschiedenen Teilen des Landes in der zweiten Hälfte des neunzehnten Jahrhunderts nicht als positive Merkmale hervor. Das war nicht nur darauf zurückzuführen, daß sie im Fortschritt den Zerstörer der regionalen Unterschiede sahen, sondern auch darauf, daß sie noch an die Ideale der Einheitsbewegung glaubten. Ein Wissenschaftler wie Pitrè, der sich freiwillig für die Armee Garibaldis verpflichtete, verstand die Sizilianität nur als ein Kulturerbe, das über kurz oder lang in der nationalen Kultur aufgehen und mit ihr verschmelzen würde. ${ }^{9}$

Um die Jahrhundertwende förderten ein wirtschaftlicher Aufschwung und aufkeimende sozialen Konflikte das Wiederauftauchen der regionalen Problematik sei es als politisches Reformprojekt, sei es als Identitätsproblem. Die Notwendigkeit, ökonomisch die Stadtgrenzen zu überwinden, bewog dazu, provinziale und regionale Industrie- und Handelsmessen zu veranstalten. In ihrem Umfeld und in diesem Klima wurden aber neue regionale Stereotypen verbreitet. 1882 hatte Turiello die Einheit des italienischen Charakters betont: „Das Neapolitanische“ unterscheide sich davon beispielsweise nur graduell. Um die noch bestehenden Differenzen zwischen Nord und Süd zu erklären, bildete jetzt die positivistische Literatur Interpretationen, die sich auf eine biologistische Basis stützten. 1901 stellte Alfredo Niceforo eine arische Rasse im Norden einer mediterraneischen mit ganz bestimmten Unterschieden in den Eigenschaften gegenüber. ${ }^{10}$

An der neuen Bewertung der regionalen Kultur nahmen meistens lokale Intellektuelle teil, die die Ausbreitung der Kulturindustrie marginal zu machen suchten. Die Verherrlichung der Region war so mit eine Reaktion auf und gegen die kosmopolitische Kraft der Kulturindustrie.

In den Jahren vor dem ersten Weltkrieg, das sogennante „Età giolittiana“ führte das nationalistische Klima zwar zur Verweigerung des politischen Partikularismus,

7 TURIELlo, Pasquale: Governo e governati. Bologna 1882. S. 17.

${ }^{8}$ GRIBAUDI, Gabriella: A Eboli. Il mondo meridionale in cent'anni di trasformazione. Venezia 1990; RomANELLI, Raffaele: Le radici storiche del localismo italiano. In „Il Mulino“ J. XL H. 336 (Juli August 1991).

${ }^{9}$ COCCHIARA, Giuseppe: Pitrè la sicilia e il folklore. Firenze 1951, S. 17-18.

${ }^{10}$ NICEFORO, Alfredo: Italiani del Nord e Italiani del Sud. Torino 1901, S. $22-23$. 
aber gleichzeitig auch zu einer Wiederentdeckung der Region als eines Schrittes hin zu einem nationalen Organismus. Dazu trugen der Einfluß von französischen Autoren, insbesondere von Hippolite Taine mit seiner Milieu-Theorie, und die italienischen Geographen, die die Region als Gebiet von sogenannten natürlichen Grenzen klar bestimmten, bei. Die Konstruktion eines regionalen kulturellen Feldes fand im Bereich von lokalen Zeitschriften statt, die in dieser Zeit zahlreich entstanden. Intellektuelle aus den Provinzen widmeten sich der Untersuchung der lokalen Geschichte, der Bewertung und Pflege von Denkmälern und der Wiederentdeckung der regionalen Landschaft. Damit wurden sukzessive Elemente zur Bildung einer kulturellen Identität der Region geliefert. Natürlich formierte sich der Leserkreis zum größten Teil aus den klein-bürgerlichen Schichten; aber im Fall von satirischen und Mundart-Publikationen waren auch die Unterschichten einbezogen. Das lokale Bewußtsein wurde auch durch Dialektpoesie und Mundart-Theater, die damals ihre hohe Zeit erlebten, sowie durch Stadtfeste verbreitet.

Die Region stieß auch im volkskundlichen Bereich auf Anklang. Schon 1894 stellte Angelo de Gubernatis dem Monarchie-loyalen Protest der sizilianischen Bauern die atheistisch-subversiven Arbeiterunruhen in der Toskana gegenüber: Der erste war positiv, letztere waren negativ besetzt. Gleichzeitig forderte er aber den Gesetzgeber auf, die Vielfältigkeit des Landes zu beachten. ${ }^{11}$ Seit 1911 gaben Giovanni Podenzana und Giovanni Sittoni das „Archivio per l'etnografia e la psicologia della Lunigiana" heraus, eine Zeitschrift, die die Forschung auf eine eng umgrenzte Zone Liguriens konzentrierte. Die Auswahl eines Forschungsfeldes von subregionaler Bedeutung stand in keinem Gegensatz zur regionalen Identität, sondern wurde auf die Notwendigkeit zurückgeführt, gründliche Studien zu betreiben. ${ }^{12}$ Nach dieser Ansicht trugen historische Ereignisse und geographische Elemente zur Bestimmung des Regionalcharakters bei:

„Veränderungen des Charakters sind im Einklang mit denjenigen der Umgebung, und da diese hier schroff sind [...], ergibt sich konsequenterweise eine Disharmonie des Charakters, die „Die Lunigiana“ insgesamt charakterisiert". ${ }^{13}$

In demselben Jahr wurde anläßlich des fünzigsten Jahrestages der italienischen Einigung eine ethnographische Ausstellung organisiert, die u.a. nach regionalen Kriterien gegliedert war. Die regionale Problematik trat auch im ersten ethnographischen Kongreß stark hervor. Dort wurde entschieden, daß das geplante nationale

${ }^{11}$ DE GUBERnATIS, Angelo: Le sommosse italiene ed il folk-lore. In „Rivista delle tradizioni popolari italiane“ J. I (1894), S. 171-172. Darüber vgl. auch PUCCINI, Sandra: Introduzione. In PuCCINI, Sandra (Hrg.): L'uomo e gli uomini. Scritti di antropologi italiani dell'Ottocento. Roma 1991, S. 34ff.

12 Sittoni, Giovani-PodenZanA, Giovanni: Concetto e tema del nostro Archivio. In: „Archivio per l'etnografia e la psicologia della Lunigiana“. J. I (1911), S. 4.

13 G.S.: Piccole communicazioni. Per un atlante etnografico della Lunigiana. In: „Archivio per l'etnografia e la psicologia della Lunigiana“. J. I (1911), S. 49. 
Museum einer regionalen Ordnung folgen müßte. ${ }^{14}$ Obwohl Francesco Baldassaroni auf den Unterschied zwischen geographischen und ethnographischen Regionen verwiesen hatte, ${ }^{15}$ war der Tenor der Tagung ein Zeichen für das neue Klima. Im folgenden Jahr betonte Lamberto Loria die Rolle der Ethnographie nicht nur als Hilfe für die koloniale, sondern auch für die innere Politik. Die Volkskunde konnte so einerseits eine bessere Kenntnis der südlichen Regionen, andererseits Elemente zu einer Gesetzgebung liefern, die die regionale Verschiedenheit des Landes anerkennen konnte. ${ }^{16}$ Die Rolle der regionalen Kulturen wurde 1914 in einem Werk von Giovanni Crocioni klar theoretisiert. Crocioni plädierte für die Einführung regionaler Heimatkunden als Schulfächer. Nach seiner Auffassung waren die kulturellen Merkmale das Ergebnis von Boden, Rasse, Milieu und Geschichte. Infolgedessen wa-ren die regionalen Charaktere eine langfristige Erbschaft, die bis in die römische Zeit zurückreichte. Beeinflußt von Taine sowie von der geographischen Schule und von pädagogischen Traditionen, stand Crocionis Bewertung der Region überhaupt im Einklang mit einer patriotischen Auffassung: Über den Umweg der Region beabsichtigte er die Bildung einer nationalen Identität für die Italiener. ${ }^{17}$

Im ersten Weltkrieg führten die Mobilmachung und der Alltag im Schützengraben mit seinem Zusammentreffen unterschiedlicher regionaler Kulturen zu einer Stärkung der Nationalisierungsbestrebungen. Trotzdem wurde die Wiederentdeckung der Region nicht gestoppt, sondern von der Krise der Nachkriegszeit begünstigt. Die Bewertung der Region war aber stark von patriotischen Ideen geprägt. In den zwanziger Jahren versuchte der Faschismus, durch die Verwendung der regionalen Kultur das nationale Bewußtsein zu stärken. ${ }^{18} 1923$ wurden Dialekte und Regionalkulturen in die Unterrichtsprogramme der Schulen eingeführt. Ab 1927 veranstaltete das Dopolavoro - die Freizeitorganisation des Regimes - Feste und gründete Chöre und Mundartvereine, die regionale und städtische Ideologien forcierten. Einige Intellektuelle hofften sogar auf eine Neubegründung der nationalen Kultur auf regionaler Basis im Gegensatz zu den modernen kosmopolitischen Tendenzen. In der Zeitschfrit „Scuola fascista“ (faschistische Schule) liest sich das so:

14 Verbali delle sedute. In: Atti del I Congresso di etnografia Italiana (Roma 19-24 Ottobre 1911). Perugia 1912, S. 45-53. Über die italienische Ethnologie in dieser Zeit vgl. PUCCINI, Sandra: Evoluzioismo e positivismo nell'antropologia italiana (1896-1911). In CLEMENTE, Pietro et al: L'antropologia italiana: un secolo di storia. Roma-Bari 1985, S. 97-148.

15 BALDASSERONI, Francesco: Il museo di etnografia italiana. Coordinamento in regioni o per categorie di oggetti. in: „Lares. Bollettino della società di etnografia italiana“. J. I (1912), H. I, S. 44 und p. 50. Über die Geschichte der Ethologie vgl. zusammenfassend CIRESE, Alberto Mario: Cultura egemonica e culture subalterne. Palermo 1980, S. 165ff.; vgl. auch AA. VV: Studi antropologici italiani e rapporti di classe dal positivismo al dibattito attuale. Milano 1980.

16 LORIA, Lamberto: L'etnografia strumento di politica interna e coloniale. In: „Lares. Bollettino della società di etnografia italiana“" J. I (1912), H. I, S. 78-79.

${ }^{17}$ CROCIONI, Giovanni: Le regioni e la cultura nazionale. Catania 1914.

18 Darüber vgl. CAVAZZA, Stefano: Tradizione regionale e riesumazioni demologiche. In: „Studi Storici“ J. 34 H. 2-3 (Apr.-Sept. 1993), pp. 638-640; CAVAZZA, Stefano: Piccole patrie. Feste popolari tra regione e nazione durante il fascismo. Bologna 1997, S. 95 ff. 
„Die Region ist die Grundlage aller unserer Neigungen, die uns artgemäß sind; sie ist Summe aller Erlebnisse derer, die vor uns lebten [...]. Wir sind wie Pflanzen: unsere Wurzeln erlauben uns, uns zu bewegen, ja sogar umzusiedeln, aber sie erlauben uns nicht, unsere Herkunft zu verleugnen. [...] wir tragen immer und überall das Gefühl und das Gewissen der Heimat in uns".$^{19}$

Auf jeden Fall schloß die faschistische Bewertung der Region jede regionale Autonomie aus und strebte die Überwindung der Region an.

Obwohl in den dreißiger Jahren das Regime eine Kampagne gegen Mundart und Region unternahm, ging die Verwendung von lokalen Kulturen weiter - etwa im Bereich der Freizeitorganisationen. Die faschistische Wende hatte aber ihre Folgen: Einerseits wurden die gemeinsamen Elemente der verschiedenen regionalen Kulturen und die römische Herkunft noch stärker betont, andererseits wurde oft ein städtischer Patriotismus dem regionalen vorgezogen.

Im bisher geschilderten Bild nahm die Romagna einen wichtigen Platz ein, weil die Romagna auf eine starke regionale Tradition verweisen konnte, obwohl sie niemals Verwaltungsgebiet war. Der Name Romània bezeichnete die Gebiete, die außerhalb der langobardischen Herrschaft lagen: das sogenannte byzantinische Exarchat. Die eindeutige Bestimmunmg der regionalen Grenzen geschah aber sehr spät. Noch 1841 bezog Antonio Vesi Bologna und Ferrara in die Romagna ein - aufgrund der historischen Tradition, der geographischen Form, des einheitlichen Charakters und des Dialektes. ${ }^{20}$ Erst im Jahre 1894 beschränkte Emilio Rosetti die Romagna auf die Provinzen von Ravenna und von Forli und auf Teile der Provinzen von Florenz, von Bologna und von Pesaro - eine Einteilung, die der aktuellen entspricht. ${ }^{21}$ Aber noch 1928 vertrat Vittorio Cian die Idee, daß die Romagna auch Bologna und Ravenna einbeziehen müsse. ${ }^{22}$

Im Lauf der Zeit hatte sich ein Stereotyp des „Romagnolo“ entwickelt: impulsiv, rachgierig, gewalttätig. In dieses Bild fügten sich die Romagnoli schon im 16. und 17. Jahrhundert ein. ${ }^{23}$ Nach den Prozeßakten vor dem Kriminalgericht in Bologna habe der Graf Battaglini seinen Diener Ludovisi etwa im Jahre 1740 gedroht, ,den Stock und die Büchse reden zu lassen, wie das die Romagnolen tun"..24 Das Stereotyp wurde auch im neunzehnten Jahrhundert verbreitet, um die Aufstände gegen die päpstliche Regierung und die Kriminalakte zu erklären. Nach der Vereinigung wurde dann der harte soziale Protest auf die Primitivität der Romagnolen zurück-

${ }^{19}$ AmorosA, Berengario: Il Libro regionale. In: Scuola Fascista. H. 50, 25 Mai 1928.

${ }^{20}$ VESI, Antonio: Ragionamento intorno ai veri confini di Romagna. Faenza, Montanari e Marabini, 1841

${ }^{21}$ GAMBI, Lucio: La „Romagna“ di Emilio Rosetti. In: „Romagna arte e storia“ J.X H. 30 (Sept.-Dez. 1990), S. 83 e ss.

22 CiAN, Vittorio: L'ora della Romagna. Bologna 1928.

23 Über den Stereotyp vgl. CAMPORESI, Piero: Lo stereotipo del romagnolo. In: „Studi Romagnoli“ J. XXV (1974), S. 393-411.

${ }^{24}$ Zitat nach SARTI, Raffaella: Per una storia del personale domestico in Italia: il caso di Bologna (secc. XVIII-XIX). Tesi di Dottorato di Ricerca V Ciclo, 1993, S. 166. 
geführt. Der Präfekt Giuseppe Campi sprach von ihrem „brutalen und grausamen Instinkt". Nach der Ansicht eines Grundschullehrers war die Vaterlandsliebe der Romagnolen sehr schwach. weil die Mentalität des Bauern auf einem lokalpatriotischen Kreis begrenzt war. ${ }^{25}$ Abgesehen davon, daß Kriminalität auch in anderen Gegenden verbreitet war, lagen die Ursachen allerdings in der zurückgebliebenen Entwicklung der Romagna. 1861 war die Analphabetismusrate sehr hoch, höher als die in den Nachbargebieten. ${ }^{26}$ Das war einerseits eine Folge der starren Papstherrschaft, andererseits auch der stagnierenden Wirtschaftsentwicklung. Nach der Vereinigung versuchten sich demokratisch-radikale Gruppen zu organisieren und durch politische Gesellschaften und gegenseitige Hilfeleistungsvereine die bäuerlichen Schichten zu erziehen. Die Region wurde so zum Feld einer politischen und sozialen Konfrontation. Hier entstand die consociazione repubblicana, die den Kampf von Giuseppe Mazzini gegen die Monarchie fortsetzte. ${ }^{27}$ Hier gewann auch 1882 ein Sozialist zum ersten Mal einen Sitz im Parlament. Infolge der wirtschaftlichen Modernisierung gab es neben den Halbpächtern auch immer mehr Tagelöhner. Die Agrarkonflikte wurden deshalb im zwanzigsten Jahrhundert noch härter. Sie eskalierten im Juni 1914 in der sogenannten settimana rossa (rote Woche) in der Romagna und in den Marken. Dabei handelte es sich um einen spontanen Aufstand, der zu Plünderungen und zur naiven Ausrufung einer Republik führte. ${ }^{28}$ Zur Erklärung solchen „Umstürzlertums“ wurden die alten Stereotypen wieder bemüht. Guglielmo Ferrero knüpfte schon 1894 an das Stereotyp des gewalttätigen Romagnolo an. Die Ereignisse der „Roten Woche“ haben das kollektive Gedächtnis so stark geprägt, daß in Oral History-Interviews noch heute ein Zusammenhang zwischen romagnolità und der roten Woche behauptet wird.

Seit der Jahrhundertwende wurden auch in der Romagna zahlreiche lokale Zeitschriften herausgegeben, die der Aufwertung der Region dienen sollten und in denen Intellektuelle wie Aldo Spallicci, Piero Zama und Antonio Beltramelli ein neues Stereotyp verbreiteten, das jedoch die positiven Merkmale des romagnolischen Volks betonte: Glaube, Fleiß, Opferbereitschaft, usw. Unter der Leitung von Spallicci entstand 1911 „Il Plaustro“, eine Publikation, die der Volkskunde viel verdankte. In Anlehnung u. a. an das Vorbild des provenzalischen Dichters Mistral vertrat Spallicci einen Regionalismus, der die Verbundenheit zwischen Natur und Kultur und zwischen der Gegenwart und der Vergangenheit betonte. Anläßlich einer regionalen Ausstellung sagte er:

25 PIVATO, Stefano: Pane e grammatica. L'istruzione elementare in Romagna alla fine dell'800. S. 42.

${ }^{26}$ Pivato, Stefano, Pane (Wie Anm. 25). S. 22.

27 Vgl. z. B. BALzANI, Roberto: Circoli e politica. Le origini della Consociazione Repubblicana Ravennate 1863-1872. Bologna 1993.

28 Über settimana Rossa vgl. MARTINI, Manuela: Giugno 1914. Folle romagnole in azione. In: „Rivista di storia contemporanea“ H. 4 1989, S. 517-559, über den Stereotyp S. 520-521. 
„Hier wo der römische Legionär durchgezogen ist, um die Welt zu erobern, ist die Seele seit Ewigkeit unbesiegt geblieben. Dies ist unsere Heimat. Es ist eine Heimat, gesammelt in den Dingen, die eine Seele haben". .29

In Spalliccis populistischer Auffassung spielte die Volkskunde eine wichtige Rolle, weil sie ein Zeichen des regionalen Geistes war. Spallicci versuchte, die regionale Kultur wiederzubeleben. Er verfaßte Mundartlieder, die sogar von der faschistischen Freizeitorganisation „dopolavoro“ aufgeführt wurden, obwohl Spallicci kein Faschist war und seinen Wohnort nicht verlassen durfte. Seine Dialektwerke gelten als echte romagnolische Folklore und waren übrigens kein Einzelfall. Musiker wie Francesco Balilla-Pratella, einer der Mitbegründer der futuristischen Bewegung, oder der Schriftsteller Antonio Beltramelli trugen ebenfalls zu dieser Wiederbelebung der regionalen Kultur bei. Nachdem der Plaustro sein Erscheinen eingestellt hatte, gab Spallicci 1920 eine neue Zeitschrift unter dem Titel „La Piè“ heraus..$^{30}$ Die Redaktion förderte die Entstehung von Volkschören und belebte alte Bräuche wie das Trebbo (ein traditionales bäuerliches Zusammentreffen) wieder. Der Faschismus trug zur Bestärkung des positiven Stereotyps bei. In der Romagna war Benito Mussolini geboren, so daß man nur von den positiven Merkmalen des Gebiets sprechen konnte. Einige Mitarbeiter von Spallicci, wie Piero Zama oder Balilla-Pratella traten in die faschistische Bewegung ein. Obwohl die Zeitschrift „Spallicci“ 1993 aufgelöst wurde, blieb die Wertschätzung der regionalen Kultur auch in faschistischen Publikationen bis zum Zweiten Weltkrieg erhalten. Die seit 1941 erschienene Zeitschrift „il Trebbo“ vertrat einen Regionalismus, der von irrationalistischen und chauvinistischen Elementen gekennzeichnet war:

„In Anlehnung an die alte Tradition, rufen wir [...] die erlesenen Köpfe unseres Stammes zusammen, um über die Geschichte, die Helden und die Sitten der Romagna zu reden. Indem wir unsere Region verherrlichen, verherrlichen wir gleichzeitig unsere größere gemeinsame Heimat: Italien [...] Hier werden sich die kleinen Leute in mystischer Verbindung mit den Gelehrten versammeln [...] Liebe, Großzügigkeit, Glaube werden unsere Zeitschrift „Trebbo“ leiten, während das Trebbo den Stolz überliefern wird, die Scholle dieses sonnigen Landes durch die Mühe fruchtbar zu machen und der Heimat im Krieg das Opfer des romagnolischen Blutes zu erbringen. “31

\footnotetext{
29 Über Spallicci und „La piè“ vgl. CAVAZZA, Stefano: Il regionalismo in una transizione di regime: „La piè “ e l'identità culturale romagnola. In „Memoria e Ricerca“ N.F. H. 2 (1998), S. 77-99.

30 Über die Geschichte der Zeitschrift vgl. CASALI, Elide, La Piè e la cultura folclorica romagnola durante il fascismo. In: BATTISTINI, Andrea (Hrg.) Aspetti della cultura emiliano-romagnola nel ventennio fascista. (= Istituto regionale Ferruccio Parri per la storia del movimento di liberazione e dell'età contemporanea in Emilia Romagna. Annale 9) Milano 1992, S. 239-293.

${ }^{31}$ N.G. In: Il Trebbo. J. II H. 1 Juni 1942, S. 1.
} 
Das Beispiel der Romagna zeigt, daß sich die Konstruktion der kulturellen Identität stark den intellektuellen und politischen Eliten verdankte, die die alten Stereotype ins Positive kehrten. Die populistische Argumentation der Eliten begünstigte die Konstruktion eines kulturellen Feldes, in dem sich verschiedene soziale Schichten wiedererkennen konnten. Sehr interessant ist dabei, daß auch der Faschismus trotz seines zentralistischen Nationalismus nicht im Gegensatz zur regionalen Identität stand, sondern einen patriotischen Regionalismus entwickelte, dessen Wurzeln in einer behaupteten gemeinsamen Identität lagen. 